\title{
Inelastic cotunneling through a long diffusive wire
}

\author{
M. V. Feigelman and A. S. Ioselevich \\ L.D.Landau Institute for Theoretical Physics, Moscow 119334, Russia, \\ Moscow Institute of Physics and Technology, Moscow 141700, Russia.
}

(Dated: October 31, 2018)

\begin{abstract}
We show that electron transport through a long multichannel wire, connected to leads by tunnel junctions, at low temperatures $T$ and voltages $V$ is dominated by inelastic cotunnelling. This mechanism results in experimentally observed power-law dependence of conductance on $T$ and $V$, in the diffusive regime where usual Coulomb anomaly theory leads to exponentially low conductance. The power-law exponent $\alpha^{*}$ is proportional to the distance between contacts $L$.

PACS numbers: 73.63.-b, 73.23.Hk
\end{abstract}

Electronic transport through nanowires was intensively studied by many groups in the past years. In particular, conductance of multi-channel diffusive nanowires with relatively poor contacts to metal terminals was measured, cf. e.g. [1]. The Coulomb phenomena play an important role in transport, provided the contacts between the wire and the leads are weak. The mechanism of the Coulomb blockade, as well as the Coulomb anomaly due to tunneling spreading of charge, are presently well understood. In the ballistic regime (at relatively high temperature $T$ and/or bias voltage $V$ ) the Coulomb effects lead to the power-law temperature and voltage dependence of the conductance:

$$
G \equiv d I / d V \propto V^{\alpha}(\operatorname{low} T), \quad G \propto T^{\alpha}(\text { high } T),
$$

characteristic for Luttinger Liquid, while at low $T$ and $V$ - in diffusive regime - an exponential dependence (see (44) below) should be observed. The puzzle is that the power law (1) is found in almost all experiments, even in those where the conditions for the diffusive regime seem to be fulfilled.

The existing theories (see, e.g., 2], 3], [4]) considered the Coulomb effects at each of contacts separately. However, if both contacts are taken into account simultaneously, then some analog of cotunneling becomes possible and at low temperatures this mechanism should dominate. The standard theory of cotunneling deals with small grains or quantum dots, while a long wire is an extended object: internal dynamics of charge within it may be important. In the present letter we develop a theory for such an extended cotunneling and show that in the diffusive regime the resulting cotunneling conductance still obeys the law (11), though with different exponent $\alpha^{*}$, depending on the separation $L$ between the contacts.

Consider a multichannel metallic wire (it may be a multiwall nanotube) of length $L_{0}$ and diameter $a$. The wire is connected to massive metallic leads through two weak tunnel contacts $A$ and $B$ with identical dimensionless conductances $g \ll 1$ (see Fig,1). A voltage, applied between the contacts is $V$. The classic dimensionless resistance of the piece of wire between the leads is assumed to be not very small: $R(L) /\left(h / e^{2}\right) \equiv L / \xi \gtrsim 1$, where

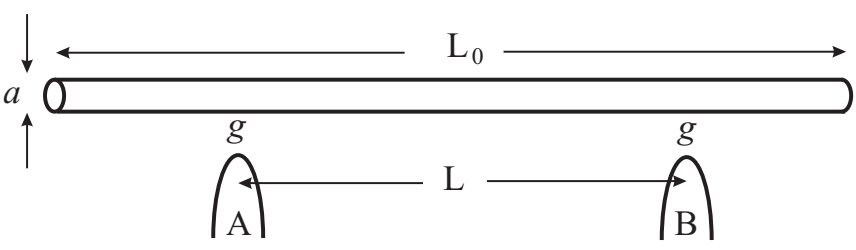

FIG. 1: Electrons tunnel between a wire of length $L_{0}$ and diameter $a$ and two leads $A$ and $B$, placed symmetrically with respect to the center of the wire, at distance $L$ from each other.

$\xi \sim N_{\text {ch }} l$ is the localization length, $l$ is the mean free path, and $N_{\mathrm{ch}} \gg 1$ is the number of channels.

In this paper the relevant energy scales will be assumed so low, that the motion of electrons in the wire is diffusive. On the other hand, we will neglect the localization effects. As long as usual conductivity in a wire is concerned, the condition of "no localization" reads

$$
T \gg T_{\mathrm{Loc}} \sim D \xi^{-2} \sim v_{F} / N_{\mathrm{ch}}^{2} l .
$$

It is not evident that inequlity (2) is in fact necessary when the under-the-barrier spreading process is considered; however, it is certainly the sufficient one, and we will assume it is fulfilled below. This requirement is consistent at $N_{\mathrm{ch}} \gg 1$ with the diffusive dynamics of charge spreading.

If the temperature $T$ is not very low, the diffusive transport between the two leads proceeds in a "singleparticle mode": At first one electron (one hole) tunnels into the wire from one of the leads and is accommodated in the wire, then one hole (one electron) tunnels from another lead. Because of the (thermoactivated) tunneling character of the accommodation process, the corresponding conductance $G_{A B}^{(1)}$ is exponentially suppressed

$$
G_{A B}^{(1)} \sim g \exp \left\{-S_{1}(T, V)\right\}
$$

At temperatures $T \gg T_{c}^{(1)} \equiv \frac{E_{C}^{2}\left(L_{0}\right)}{E_{C}(\xi)}$ the accommodation proceeds according to the semiclassic scenario 2 (the Coulomb zero-bias-anomaly regime, see also [3, [5, [6]), 
and the accommodation action

$$
S_{1}(T, V) \approx\left\{\begin{array}{cl}
0.76 \sqrt{\frac{E_{C}(\xi)}{T}}, & \text { for } e V \ll \sqrt{E_{C}(\xi) T}, \\
E_{C}(\xi) /(e V), & \text { for } e V \gg \sqrt{E_{C}(\xi) T} .
\end{array}\right.
$$

Here $E_{C}(x)=e^{2} \ln (x / a) / \epsilon x$ is the charging energy of a piece of wire of length $x \gg a$.

At $T \ll T_{c}^{(1)}$ the single-particle accomodation proceeds according the "orthodox" Coulomb blockade scenario (see, e.g., 7] ):

$$
S_{1}(T, V) \approx E_{C}\left(L_{0}\right) / T .
$$

The abovementioned independent single particle processes should be less effective than some correlated cotunneling process, in which any charged states of the wire would only enter as virtual intermediate states. The theory of such processes is well developed for transitions via small grains, where the intergrain charge transfer processes are the bottlenecks for the transport, while the intragrain charge transfer is easy (see [8]). In our case, however, the charge spreading within the wire is a crucial ingredient of the process, so that the standard perturbational description of the cotunneling is inapplicable.

In the present Letter we propose a modification of the approach [2], which allows for description of charge spreading effects under the two-particle cotunneling conditions. Our main result reads as follows:

$$
G_{A B}^{(2)} \sim g^{2}\left(\frac{\max \{L T / \xi,(e V)\}}{E_{C}(L)}\right)^{\alpha^{*}}, \quad \alpha^{*}=\frac{R(L)}{\left(h / 2 e^{2}\right)},(6)
$$

In the case $L \approx L_{0}$ the crossover from one-particle tunneling (in the Coulomb blockade mode) to the two-particle one takes place at

$$
T_{c}^{(2)} \sim \frac{e^{2} \xi}{\epsilon L_{0}^{2}} \frac{\ln \left(L_{0} / a\right)}{\ln \left(\frac{e^{2} \xi}{\epsilon L_{0}^{2} T}\right)+\frac{\xi}{L_{0}} \ln (1 / g)}<T_{c}^{(1)} .
$$

The last inequality becomes strong for very low conductance of contacts, $g \ll 1$; in this situation a sequence of crossovers may be seen with the temperature decrease: from the Coulomb anomaly mode (44) to the Coulomb blockade mode (5) at $T=T_{c}^{(1)}$, and then to the inelastic cotunneling regime (6) at $T=T_{c}^{(2)}$.

In the case $L \ll L_{0}$ the Coulomb blockade regime is absent, and the crossover from the Coulomb anomaly to the inelastic cotunneling takes place at

$$
T_{c}^{(2)} \sim \frac{e^{2} \xi}{\epsilon L^{2}} \frac{\ln (\xi / a)}{\left[\ln \left(\frac{e^{2} \xi}{\epsilon L^{2} T}\right)+\frac{\xi}{L} \ln (1 / g)\right]^{2}} .
$$

In the nonlinear regime the crossover between the singleparticle Coulomb anomaly and the inelastic cotunneling takes place at

$$
e V \sim(e V)_{\mathrm{cr}} \approx \frac{e^{2}}{\epsilon L} \ln (\xi / a)
$$

Thus, at low enough temperatures, the cotunneling scenario always dominates. On the other hand, the condition (2) of "no localization" should also be fulfilled for applicability of the formula (6). The necessary temperature range only exists if

$$
1 \lesssim \frac{L}{\xi} \ll \sqrt{\frac{E_{C}(\xi)}{T_{\mathrm{Loc}}}} \sim\left[\frac{\ln (\xi / a)}{\epsilon} N_{\mathrm{ch}}\right]^{1 / 2} .
$$

i.e. the condition $N_{\mathrm{ch}} \gg 1$ is necessary.

The method of Levitov and Shytov [2] is based on classic equations of motion for the electron density $\rho(x, \tau)$ and current $j(x, \tau)$ in imaginary time $\tau=-i t$. In a case of wire one can write

$$
\begin{array}{r}
\frac{\partial \rho}{\partial \tau}+i \frac{\partial j}{\partial x}=\mathcal{J}(x, \tau), \\
j+D \frac{\partial \rho}{\partial x}-\tilde{\sigma} \frac{\partial}{\partial x} \int d x^{\prime} \rho\left(x^{\prime}, t\right) \frac{1}{\epsilon\left|x-x^{\prime}\right|},
\end{array}
$$

where $\tilde{\sigma}=e^{2} \xi / 2 \pi \hbar$ is effective one-dimensional conductivity, $\epsilon$ is the effective dielectric constant, and $D$ is a diffusion constant. The instanton is chosen in a form of a symmetric bounce, so that the source $\mathcal{J}$ in the continuity equation (11) corresponds to the injection of one extra electron into the system at time $t_{1}=-i \tau_{0}$ at point $x=$ $-L / 2$ with its subsequent elimination at the same point at moment $t_{2}=i \tau_{0}: \mathcal{J}_{1}=\left[\delta\left(\tau+\tau_{0}\right)-\delta\left(\tau-\tau_{0}\right)\right] \delta(x+L / 2)$.

The crucial point of our approach is that we describe contunnelling through a diffusive wire by the same semiclassical equations (1112), but with modified source

$$
\mathcal{J}_{2}=\left[\delta\left(\tau+\tau_{0}\right)-\delta\left(\tau-\tau_{0}\right)\right][\delta(x+L / 2)-\delta(x-L / 2)]
$$

which describes simultaneous tunnelling of an electron and a hole via both contacts.

The density $\rho(x, \tau)$ should be real and even with respect to $\tau \rightarrow-\tau$, while the current $j(x, \tau)$ should be purely imaginary and odd. $\rho(x, \tau)$ and $j(x, \tau)$ are defined on the interval $-1 / 2 T<\tau<1 / 2 T$ and obey periodic boundary conditions. Expanding $\rho(x, \tau)$ and $j(x, \tau)$ in Fourier series, we get $\rho(x, \tau)=\sum_{\omega} \rho(x, \omega) \cos (\omega \tau)$ and $j(x, \tau)=\sum_{\omega} j(x, \omega) \sin (\omega \tau)$, where the Matsubara frequency summation, as usual for Bose excitations, runs over even frequencies $\omega=2 \pi T n$, with $n=0,1,2, \ldots$.

If the wire is very long $\left(L_{0} \rightarrow \infty\right)$, the system of equations (1112) can be solved by the spatial Fourier transformation. Then, proceeding in the full analogy with [2], we obtain $G_{A B}^{(2)} \approx g^{2} \exp \left\{-S_{2}(T, V)\right\}$, where

$$
\begin{array}{r}
S_{2}(T, V)=\tilde{S}_{2}\left(T, \tau_{0}^{*}\right)-2 e V \tau_{0}^{*}, \\
\partial \tilde{S}_{2}\left(T, \tau_{0}\right) /\left.\partial \tau_{0}\right|_{\tau_{0}=\tau_{0}^{*}}=2 e V .
\end{array}
$$




$$
\begin{array}{r}
\tilde{S}_{2}\left(T, \tau_{0}\right)=\frac{e^{2}}{2} \sum_{\omega, q} \frac{\left|\mathcal{J}_{2}(\omega q)\right|^{2} U_{q}}{\left(\omega+D q^{2}\right)\left(\omega+\tilde{\sigma} q^{2} U_{q}\right)}, \\
\mathcal{J}_{2}(\omega, q)=-4 i \sin \left(\omega \tau_{0}\right) \sin (q L / 2), \\
U(q) \equiv \operatorname{reg} \int_{-\infty}^{\infty} \frac{e^{i q x}}{\epsilon|x|} d x=\frac{2}{\epsilon} \ln \frac{1}{q a} .
\end{array}
$$

The semiclassical method, used above, is applicable, if $\tilde{S}_{2} \gg 1$. From (16[17) it is clear, that $\partial \tilde{S}_{2} / \partial \tau_{0}=0$ for $\tau_{0}=1 / 4 T$. Therefore we conclude that

$$
\tau_{0}^{*}(T, V \rightarrow 0)=1 / 4 T,
$$

so that in the low-voltage case the summation runs only over the odd $n=2 k+1$ :

$$
\sum_{k=0}^{\infty} \int \frac{d q}{2 \pi} \frac{S_{2}(T, V \rightarrow 0) \approx}{\left(2 \pi T(2 k+1)+D q^{2}\right)\left(2 \pi T(2 k+1)+\tilde{\sigma} q^{2} U_{q}\right)}
$$

There are three different temperature ranges: $T \gg \omega_{\max }$; $\omega_{\min } \ll T \ll \omega_{\max } ;$ and $T \ll \omega_{\min }$, where

$$
\begin{array}{r}
\omega_{\max }=\tilde{\sigma} L^{-2} U\left(L^{-1}\right) \sim(\xi / L) E_{C}(L), \\
\omega_{\min } \sim D L^{-2} \sim \omega_{\max }\left[N_{\mathrm{ch}} \frac{\ln (L / a)}{\epsilon}\right]^{-1} \ll \omega_{\max },
\end{array}
$$

we consider these three ranges separately

1. $T \gg \omega_{\max }$. Here the sum is dominated by $k \sim 1$, $\omega \sim T$; it can be shown that $S_{2}(T, V \rightarrow 0) \approx 2 S_{1}(T, V \rightarrow$ $0)$, which means that in this temperature range the two-particle process looses a competition with the oneparticle Coulomb anomaly one.

2. $\omega_{\min } \ll T \ll \omega_{\max }$. Here the integral over $q$ is dominated by $q \sim L^{-1}$, while the logarithmical sum over $k$ is dominated by an interval $0<k \ll \omega_{\max } / T$, so that

$$
S_{2}(T, V \rightarrow 0) \approx \frac{2 L}{\xi} \ln \left(\frac{\omega_{\max }}{T}\right),
$$

and, with the help of (20), we arrive at the final expres$\operatorname{sion}(6)$.

3. $T \ll \omega_{\min }$. In this range presumably the elastic cotunneling should dominate. However, since $\omega_{\min } \ll$ $T_{\text {Loc }}$, the localization effects may be important here, and we do not discuss this regime.

In the case of high voltage one has $\tau_{0}^{*} \ll 1 / T$, so that the infrared cutoff of the frequency summation in (16) is $1 / \tau_{0}$ instead of $T$. As a result, we arrive at the same three regimes, but with substitution $T \rightarrow 1 / \tau_{0}$. In particular, in the most interesting regime 2 :

$$
\tilde{S}_{2}\left(T, \tau_{0}\right) \approx \frac{2 L}{\xi} \ln \left(\omega_{\max } \tau_{0}\right), \quad \tau_{0}^{*}=\frac{4 L(e V)}{\xi},
$$

and we arrive at the high-voltage version of formula (6).

In the case of a finite wire the straightforward Fourier analysis of the problem is impossible, and one has to be more accurate. It is important that, for two-particle tunneling, the total charge of the wire is always zero, so that one does not have to take into account the effects of the total charge which otherwise would be important in a finite system at low temperature. This simplification allows one to follow the lines of the solution described above, with a substitution of the Fourier analysis by decomposition over a set of eigenfunctions of a certain linear operator.

We present the action in a general form

$$
\begin{array}{r}
\tilde{S}_{2}=\frac{e^{2}}{2} \sum_{\omega}\left\{-\frac{j \cdot j}{\tilde{\sigma} \omega}+\rho \hat{U} \rho\right\}= \\
=\frac{e^{2}}{2} \sum_{\omega} \mathcal{J}_{2}\left\{-\frac{\tilde{\sigma}}{\omega} \hat{\mathcal{G}}^{+} \hat{U} \hat{\nabla}^{T} \hat{\nabla} \hat{U} \hat{\mathcal{G}}+\hat{\mathcal{G}}^{+} \hat{U} \hat{\mathcal{G}}\right\} \mathcal{J}_{2}= \\
=\frac{e^{2}}{2} \sum_{\omega} \frac{4 \sin ^{2}\left(\omega \tau_{0}\right)}{\omega} J_{2} \hat{\mathcal{G}}^{+} \hat{U} J_{2},
\end{array}
$$

$\rho=\hat{\mathcal{G}} \mathcal{J}_{2}, \quad j=\tilde{\sigma} \hat{\nabla} \hat{U} \rho=\tilde{\sigma} \hat{\nabla} \hat{U} \hat{\mathcal{G}} \mathcal{J}_{2}, \quad \hat{\mathcal{G}}=[\omega-\tilde{\sigma} \hat{\Delta} \hat{U}]^{-1}$

$$
\hat{\Delta} \equiv \frac{d^{2}}{d x^{2}}, \quad[\hat{U} \rho](x)=\operatorname{reg} \int_{-L_{0} / 2}^{L_{0} / 2} \frac{\rho\left(x^{\prime}\right) d x^{\prime}}{\epsilon\left|x-x^{\prime}\right|},
$$

The rules for regularization of the singular integral (25) are similar to those, given in [9]. Within the logarithmic accuracy one can write $[\hat{U} \rho](x) \approx U(x) \rho(x)$, where

$$
\begin{aligned}
U(x) \equiv \frac{1}{\epsilon}\left\{\ln \left[\frac{2}{a} \min \{(L / 2-x), \lambda(x)\}\right]+\right. \\
\left.+\ln \left[\frac{2}{a} \min \{(L / 2+x), \lambda(x)\}\right]\right\},
\end{aligned}
$$

and $\lambda(x) \sim[d \ln (\rho(x)) / d x]^{-1}$ is a characteristic scale of spatial variations of the function $\rho(x)$. The formula (26) is applicable, if the arguments of both logarithms are large. At $\lambda \gtrsim L_{0}$ (26) coincides with the result in [9].

Let us introduce a set of normalized righteigenvectors $\varphi_{m}(x)$ and eigenvalues $v_{m}$ of the (nonHermitean!) operator $\hat{U} \hat{\Delta}$, obeying the boundary conditions $d \varphi /\left.d x\right|_{x= \pm L_{0} / 2}=0$, corresponding to a vanishing electric field at the ends of the wire. The equation and the normalization condition for the eigenfunctions read

$$
\begin{array}{r}
\hat{\Delta} \varphi_{m}(x)=v_{m} \hat{U}^{-1} \varphi_{m}(x), \\
\int_{-L_{0} / 2}^{L_{0} / 2} \varphi_{m}(x) \hat{U}^{-1} \varphi_{m^{\prime}}(x) d x=\delta_{m m^{\prime}} .
\end{array}
$$

There is only one zero eigenvalue $v_{0}=0$, the corresponding eigenmode $\varphi_{0}(x)=$ const describes the equipotential distribution of charge in the wire. For the nonzero modes $(m=1,2, \ldots)$ in the leading logarithmic approximation

$$
\begin{array}{r}
\varphi_{m}(x)=\sqrt{\frac{2 U_{m}(0)}{L_{0}}} \cos \left[\frac{\pi m}{2}+\frac{\pi m x}{L_{0}}\right], \\
v_{m}=-\pi^{2} m^{2} U_{m}(0) / L_{0}^{2},
\end{array}
$$


where $U_{m}(x)$ is given by (26) with $\lambda(x)=L_{0} /(m+1)$. Then, for Hermitean operator $\hat{\mathcal{G}}^{+} \cdot \hat{U}$, one has

$$
\left[\hat{\mathcal{G}}^{+} \cdot \hat{U}\right]\left(x, x^{\prime}\right)=\sum_{m} \frac{\varphi_{m}(x) \varphi_{m}\left(x^{\prime}\right)}{\omega-\tilde{\sigma} v_{m}}
$$

so that the action can now be rewritten in the form

$$
\begin{gathered}
\tilde{S}_{2}=\frac{e^{2}}{2} \sum_{\omega, m} \frac{4 \sin ^{2}\left(\omega \tau_{0}\right)\left(J_{2} \cdot \varphi_{m}\right)^{2}}{\omega\left(\omega-\tilde{\sigma} v_{m}\right)}, \\
\left(J_{2} \cdot \varphi_{m}\right)=2 \sqrt{\frac{2 U_{m}(0)}{L_{0}}} \sin \frac{\pi m}{2} \sin \frac{\pi m L}{2 L_{0}} .
\end{gathered}
$$

Thus, only odd $m=2 p+1, p=0,1, \ldots$ are relevant, and

$$
\tilde{S}_{2}=\sum_{\omega} \frac{16 e^{2} \sin ^{2} \omega \tau_{0}}{L_{0} \omega} \sum_{p=0}^{\infty} \frac{U_{2 p+1}(0) \sin ^{2} \frac{\pi(p+1 / 2) L}{L_{0}}}{\omega+\frac{\pi^{2}(2 p+1)^{2} \tilde{\sigma} U_{2 p+1}(0)}{L_{0}^{2}}}
$$

Since $U_{m}(0)$ depends on $m$ logarithmically, one can replace $U_{m}(0) \rightarrow \bar{U} \equiv U_{\bar{m}}(0)$. Here $\bar{m}$ is the characteristic value of $m$ (or of $2 p+1$ ), corresponding to those terms in (34), that give the principal contribution to the sum. The value of $\bar{m}$ will be found a posteriori. As a result

$$
\tilde{S}_{2}=\frac{16 e^{2} \bar{U}}{L_{0}} \sum_{\omega} \frac{\sin ^{2} \omega \tau_{0}}{\omega} \sum_{p=0}^{\infty} \frac{\sin ^{2} \frac{\pi(p+1 / 2) L}{L_{0}}}{\omega+\frac{\pi^{2}(2 p+1)^{2} \tilde{\sigma} \bar{U}}{L_{0}^{2}}} .
$$

For $V \rightarrow 0$ we have again $\tau_{0}^{*}=1 / 4 T$. In the range of our interest $\left(\omega_{\min } \ll T \ll \omega_{\max }\right)$ one can neglect the term $\omega$ in the last denominator of (35) and write

$$
\begin{array}{r}
S_{2} \approx \frac{16 e^{2} L_{0}}{\pi^{3} \tilde{\sigma}} \sum_{k=0}^{\omega_{\max } / T} \frac{1}{2 k+1} \sum_{p=0}^{\infty} \frac{\sin ^{2} \frac{\pi(p+1 / 2) L}{L_{0}}}{(2 p+1)^{2}}= \\
=\frac{2 L}{\xi} \ln \left[\frac{\xi E_{C}(L)}{L T}\right] .
\end{array}
$$

Analyzing the series in (36), we find $\bar{m}=\overline{2 p+1} \sim L_{0} / L$. Hence

$$
\bar{U} \approx \frac{2}{\epsilon} \ln \frac{L}{a}, \quad \omega_{\max }=\frac{2 \pi^{2} \ln \frac{L}{a}}{\epsilon L^{2} \tilde{\sigma}} \sim(\xi / L) E_{C}(L) .
$$

The high-voltage modification of (36) is obtained exactly in the same way, as it was done for the case of infinite wire.

Thus, in the leading logarithmic approximation, the finite-size effects do not modify the result (6). The reason is in the special geometry, characteristic for the twoparticle cotunneling process: The initial dipole-like distribution of charge shrinks, so that the charges always move towards the center of the wire, and not in the opposite direction; therefore the presence of the ends of the wire has no effect on the process. This is not the case for one-particle tunneling, where the charge tends to proliferate equally in both directions, and the size-effect is important. These problems will be discussed elsewhere.

For interpretation of the result (6) let us think of the relevant stretch of the wire between the two contacts as a sequence of $N=L / \xi$ grains - small pieces of length $\xi$, each, connected by conductances $g_{\text {eff }} \sim 1$. Strictly speaking, the perturbation theory is only applicable, if $g_{\text {eff }} \ll$ 1 ; however, for $g_{\text {eff }} \sim 1$ it should still give qualitatively correct estimates. Then, using the results of [10], we estimate the effective conductance of this string of grains as $G_{\text {eff }} \sim g^{2}\left(g_{\text {eff }} N^{2} T^{2} / E_{C}(\xi)^{2}\right)^{N} \sim g^{2}\left(L T / \xi E_{C}(\xi)\right)^{2 L / \xi}$, which roughly agrees with (6).

In conclusion, we have shown that low-temperature diffusive transport through a wire, connected to the leads by two tunnel junctions, is realized as an inelastic cotunneling process, the effective conductance $G_{A B}^{(2)}$ obeys a power law with the $L$-dependent index. Our result (6) seems to be in agreement with the data[1]. In contrast with the ballistic case, where [3, 4] $\alpha \ll 1$ for $N_{\text {ch }} \gg 1$, our $\alpha^{*}$ can be quite large. The expression (6) for $\alpha^{*}$ is apparently similar to the formula $\alpha=Z(0) /\left(h / 2 e^{2}\right)$, used in the phenomenological "environmental theory" [11, 12]. The latter approach, however, was not able to reproduce the correct exponential behavior (3) for the single-junction setup; neither can it produce specific value $R(L)$ for the effective impedance $Z(0)$.

A financial support from the RFBR grant 06-02-16533 is gratefully acknowledged.

[1] J.-F. Dayen et al, Phys.Rev. B 72, 073402 (2005).

[2] L. S. Levitov and A. V. Shytov, JETP Letters 66, 214 (1997) and cond-mat/9607136

[3] E.G.Mishchenko, A.V.Andreev and L.I.Glazman, Phys.Rev.Lett., 87, 246801 (2001).

[4] R.Egger, A.O.Gogolin, Phys.Rev.Lett., 87, 066401 (2001).

[5] B. L. Altshuler and A. G. Aronov, in Electron-electron interactions in disordered solids, edited by A. L. Efros and M. Pollak (North-Holland, Amsterdam, 1985).

[6] A.Kamenev, A.V.Andreev, Phys.Rev. B 60, 2218 (1999).

[7] Single Electron Tunnelling, edited by H. Grabert and M. H. Devoret, (Plenum Press, New York and London, 1992).

[8] G.-L. Ingold and Yu. V. Nazarov, in Ref[7]

[9] L.D.Landau, and E.M.Lifshits, Electrodynamics of Continua, Chapter 1, Problem 9 on p.35.

[10] M.V.Feigelman, A.S.Ioselevich, JETP Letters 81, 341 (2005)

[11] A.Bachtold et al, Phys.Rev.Lett., 87, 166801 (2001).

[12] R.Tarkiainen et al, Phys.Rev., B 64, 195412 (2001). 